OPEN ACCESS

Edited by:

Marcos Egea-Cortines, Universidad Politécnica de Cartagena,

Spain

Reviewed by:

Yasser Nehela,

University of Florida, United States

Ali Parsaeimehr.

Delaware State University,

United States

*Correspondence:

Kranthi Mandadi

kkmandadi@tamu.edu

Dmitry Kurouski

dkurouski@tamu.edu

orcid.org/0000-0002-6040-4213

Specialty section:

This article was submitted to Technical Advances in Plant Science, a section of the journal

Frontiers in Plant Science

Received: 15 March 2021

Accepted: 31 May 2021

Published: 19 August 2021

Citation:

Dou T, Sanchez L, Irigoyen S, Goff N, Niraula P, Mandadi K and Kurouski D (2021) Biochemical Origin of Raman-Based Diagnostics of

Huanglongbing in Grapefruit Trees.

Front. Plant Sci. 12:680991.

doi: 10.3389/fpls.2021.680991

\section{Biochemical Origin of Raman-Based Diagnostics of Huanglongbing in Grapefruit Trees}

\author{
Tianyi Dou ${ }^{1}$, Lee Sanchez', Sonia Irigoyen ${ }^{2}$, Nicolas Goff $^{3}$, Prakash Niraula², \\ Kranthi Mandadi ${ }^{2,4 *}$ and Dmitry Kurouski ${ }^{1,5 *}$
}

\begin{abstract}
${ }^{1}$ Department of Biochemistry and Biophysics, Texas A\&M University, College Station, TX, United States, ${ }^{2}$ Texas A\&M Agrilife Research and Extension Center at Weslaco, Weslaco, TX. United States, ${ }^{3}$ Department of Biochemistry and Biophysics, Department of Biomedical Engineering, Texas A\&M University, College Station, TX, United States, ${ }^{4}$ Department of Plant Pathology and Microbiology, Texas A\&M University, College Station, TX. United States, ${ }^{5}$ Department of Biomedical Engineering, Texas A\&M University, College Station, TX, United States
\end{abstract}

Biotic and abiotic stresses cause substantial changes in plant biochemistry. These changes are typically revealed by high-performance liquid chromatography (HPLC) and mass spectroscopy-coupled HPLC (HPLC-MS). This information can be used to determine underlying molecular mechanisms of biotic and abiotic stresses in plants. A growing body of evidence suggests that changes in plant biochemistry can be probed by Raman spectroscopy, an emerging analytical technique that is based on inelastic light scattering. Non-invasive and non-destructive detection and identification of these changes allow for the use of Raman spectroscopy for confirmatory diagnostics of plant biotic and abiotic stresses. In this study, we couple HPLC and HPLC-MS findings on biochemical changes caused by Candidatus Liberibacter spp. (Ca. L. asiaticus) in citrus trees to the spectroscopic signatures of plant leaves derived by Raman spectroscopy. Our results show that Ca. L. asiaticus cause an increase in hydroxycinnamates, the precursors of lignins, and flavones, as well as a decrease in the concentration of lutein that are detected by Raman spectroscopy. These findings suggest that Ca. L. asiaticus induce a strong plant defense response that aims to exterminate bacteria present in the plant phloem. This work also suggests that Raman spectroscopy can be used to resolve stress-induced changes in plant biochemistry on the molecular level.

Keywords: Raman spectroscopy, HPLC, carotenoids, hydroxycinnamates, huanglongbing, plant biochemistry

\section{HIGHLIGHTS}

- Raman spectroscopy is coupled to high-performance liquid chromatography (HPLC) and HPLC-based mass-spectroscopy to reveal the biochemical origin of metabolic changes in citrus trees that take place upon Huanglongbing (HLB) infection. HLB causes an increase in p-coumaric acid and several flavones in the plant leaves and a decrease in the concentration of carotenoid lutein that are detected by RS. 


\section{INTRODUCTION}

Raman spectroscopy (RS) is a label-free, non-invasive, nondestructive spectroscopic technique that provides information about the chemical structure of analyzed specimens (Cardona, 1975). The Raman effect is based on inelastic scattering of photons by molecules that are being excited to higher vibrational or rotational states (Kurouski et al., 2015). RS is commonly used in food chemistry (Almeida et al., 2010), electrochemistry (Zeng et al., 2016), forensics (Kelly Virkler and Lednev, 2009; López-López et al., 2013), and materials science (Cantarero, 2015). It can be used to monitor changes in protein secondary structure (Kurouski et al., 2012), elucidate composition and origin of body fluids as well as gun-shot residues (Bueno and Lednev, 2013; Hager et al., 2018). During the last decade, several companies released portable Raman spectrometers. This allowed for the use of RS directly in the field for evaluation of plant health (Yeturu et al., 2016; Sanchez et al., 2019b,c, 2020a). This technological development sparked the interest of agronomists, plant pathologists, and plant biologists in utilization of this technology for analysis of plant health status.

The interest in agricultural applications of RS was further enhanced by a demonstration of Raman-based diagnostics of biotic and abiotic stresses. Several groups independently demonstrated that spectroscopic analysis of plant leaves and seeds could be used for confirmatory diagnostics of diseases, nitrogen, phosphorus, and potassium deficiencies as well as salinity stress in plans (Yeturu et al., 2016; Egging et al., 2018; Farber and Kurouski, 2018; Mandrile et al., 2019; Sanchez et al., 2019a,c, 2020b,c; Huang et al., 2020). Such diagnostics are based on changes in the biochemistry of the plant (Farber et al., 2019a). Moreover, it has been shown that RS could be used to identify pathogens on a species level (Egging et al., 2018; Farber and Kurouski, 2018). The potential of RS was further amplified by its coupling with chemometrics. The use of partial least square discriminant analysis (PLS-DA) and other supervised chemometric methods enabled quantitative prediction of plant diseases. On average, around $90 \%$ accurate detection and identification of plant biotic and abiotic stresses could be achieved by RS (Egging et al., 2018; Farber and Kurouski, 2018; Mandrile et al., 2019; Sanchez et al., 2019c, 2020b,c; Farber et al., 2020).

Huanglongbing (HLB) is a psyllid-vectored bacterial disease (Mcclean and Oberholzer, 1965; Capoor et al., 1967) that causes a substantial negative impact on the citrus production in Asia and Africa. Around a decade ago, HLB appeared in the Americas (Bové, 2006). The pathogen associated with this disease is currently uncultured (Tsai and Liu, 2000) Candidatus liberibacter spp. (Ca. L. asiaticus), a Gram-negative bacterium inhabits the plant phloem in uneven and variable titers, making it difficult to detect and control (Morgan et al., 2012). At early stages, trees exhibit no symptoms retaining bacteria inside the phloem. This often results in false-negative results in polymerase chain reaction (PCR)-based diagnostics of HLB. Symptomatic trees exhibit chlorotic appearance and mottling of leaves; premature drop and asymmetry of fruits (Lee et al., 2015). Relatively slow propagation of symptoms implies significant challenges in HLB management.
Typically, farmers prefer not to remove neither asymptomatic nor symptomatic trees which facilitates uncontrollable disease proliferation. Our group recently showed that RS could be used to detect and identify HLB in early and late states of the infection (Sanchez et al., 2019c). Moreover, we demonstrated that, by using RS, HLB can be distinguished from nutrient deficiency and secondary diseases such as blight (Sanchez et al., 2019b). Our recent findings show that RS is far more sensitive than qPCR, the golden standard in pathogen diagnostics (Sanchez et al., 2020d). Further elucidation of biochemical origin of RS-detected metabolite changes will further expand its utility in digital faming.

Typically, researchers assign observed spectroscopic changes to a certain Ca. L. asiaticus of chemical compounds, such as carotenoids or polyphenols (Egging et al., 2018; Farber and Kurouski, 2018; Sanchez et al., 2019c, 2020b,c; Farber et al., 2020). However, correlative understanding about the exact molecular species that is detected by Raman-based disease diagnostics is limited. Such information can be obtained by comparing RS with high-performance liquid chromatography (HPLC) and mass spectroscopy-coupled HPLC (HPLC-MS) (Hijaz et al., 2013; Killiny and Nehela, 2017; De Moraes Pontes et al., 2020). In this study, we performed systematic comparison of RS and reported HPLC (Killiny and Nehela, 2017) and HPLC-MS (Hijaz et al., 2013)-based biochemical changes associated with HLB, otherwise known as citrus greening disease.

\section{MATERIALS AND METHODS}

\section{Plants}

Leaves of HLB-positive grapefruits (Citrus $\times$ paradisi Macfad., Rio Red) without any visible signs of mechanical or insect damage were collected from the field at Texas A\&M University-Kingsville Citrus Center, Weslaco, TX $\left(26^{\circ} 10^{\prime} 00.1^{\prime \prime} \mathrm{N} 97^{\circ} 57^{\prime} 27.7^{\prime \prime} \mathrm{W}\right)$. In total, four field HLB-positive and four field HLB-negative trees were sampled and analyzed. We also collected leaves of HLB-negative trees from the greenhouse to ensure they were true negatives, and to compare their fingerprints with field healthy samples (Supplementary Figure 1). Spectroscopic analysis showed that the biochemistry of HLB-positive trees was drastically different from both healthy greenhouse-grown and field-grown trees, whereas the two healthy sets exhibited highly similar Raman fingerprints. For further comparisons, only field healthy and field HLB-positive trees were considered to keep any plant and environmental variable uniform.

\section{Isolation of DNA and Quantitative PCR (qPCR)}

Total plant DNA was isolated from the same batch of leaf samples used for carotenoid isolation. Briefly, $\sim 200 \mathrm{mg}$ tissue was homogenized in $2 \mathrm{ml}$ screw-cap microcentrifuge tubes for $60 \mathrm{~s}$ at 5,000 rpm using a Precellys 24 homogenizer (MO BIO Laboratories, Carlsbad, CA, United States). Two steel BB air gun beads (Walmart Supercenter, Bentonville, AR, United States) were added to every tube to improve homogenization (Almeyda-León et al., 2001). The final quality 

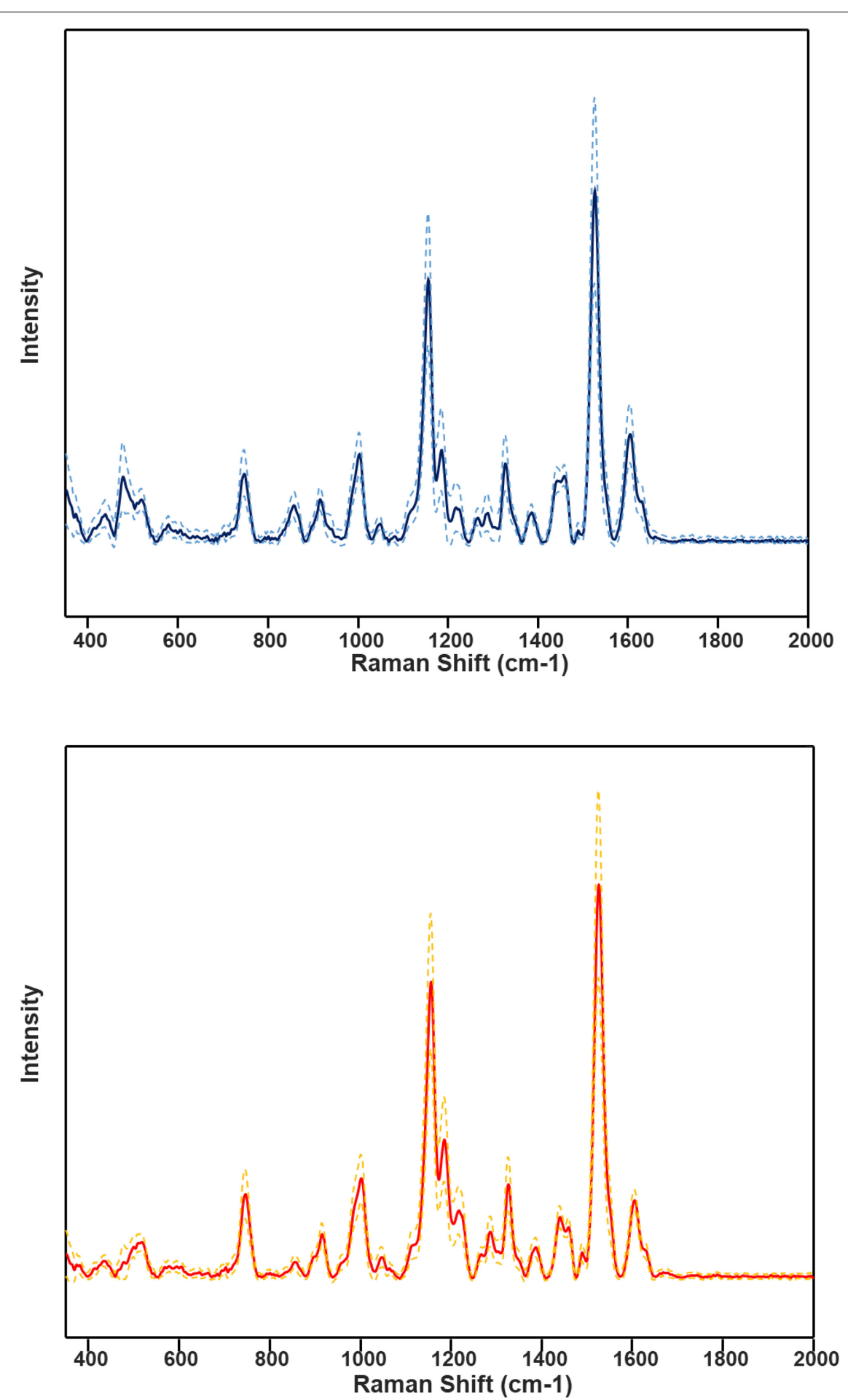

FIGURE 1 | Average HLB spectrum (blue, top) with standard deviations (dashed lines) and healthy samples (red, bottom) with corresponding standard deviations.

and quantity of DNA was measured and determined by a NanoDrop 1000 Spectrophotometer (Thermo Fisher Scientific, Wilmington, DE, United States) and agarose gel electrophoresis.

Extracted DNA was used to perform qPCR using the CFX384 Real-Time PCR Detection system (Bio-Rad Laboratories,
Inc., Hercules, CA, United States). The qPCR reaction mix contained $5 \mu$ l of iTaq universal SYBR Green Supermix (Bio-Rad Laboratories, Inc.), $0.4 \mu \mathrm{l}$ of $10 \mu \mathrm{M}$ forward primer RNR-F (5'-GGATAGTCCTGTTATTGCTCCTAAA-3'), $0.4 \mu \mathrm{l}$ of $10 \mu \mathrm{M}$ reverse primer RNR-R (5'-ACAAAGCAGAAATA 


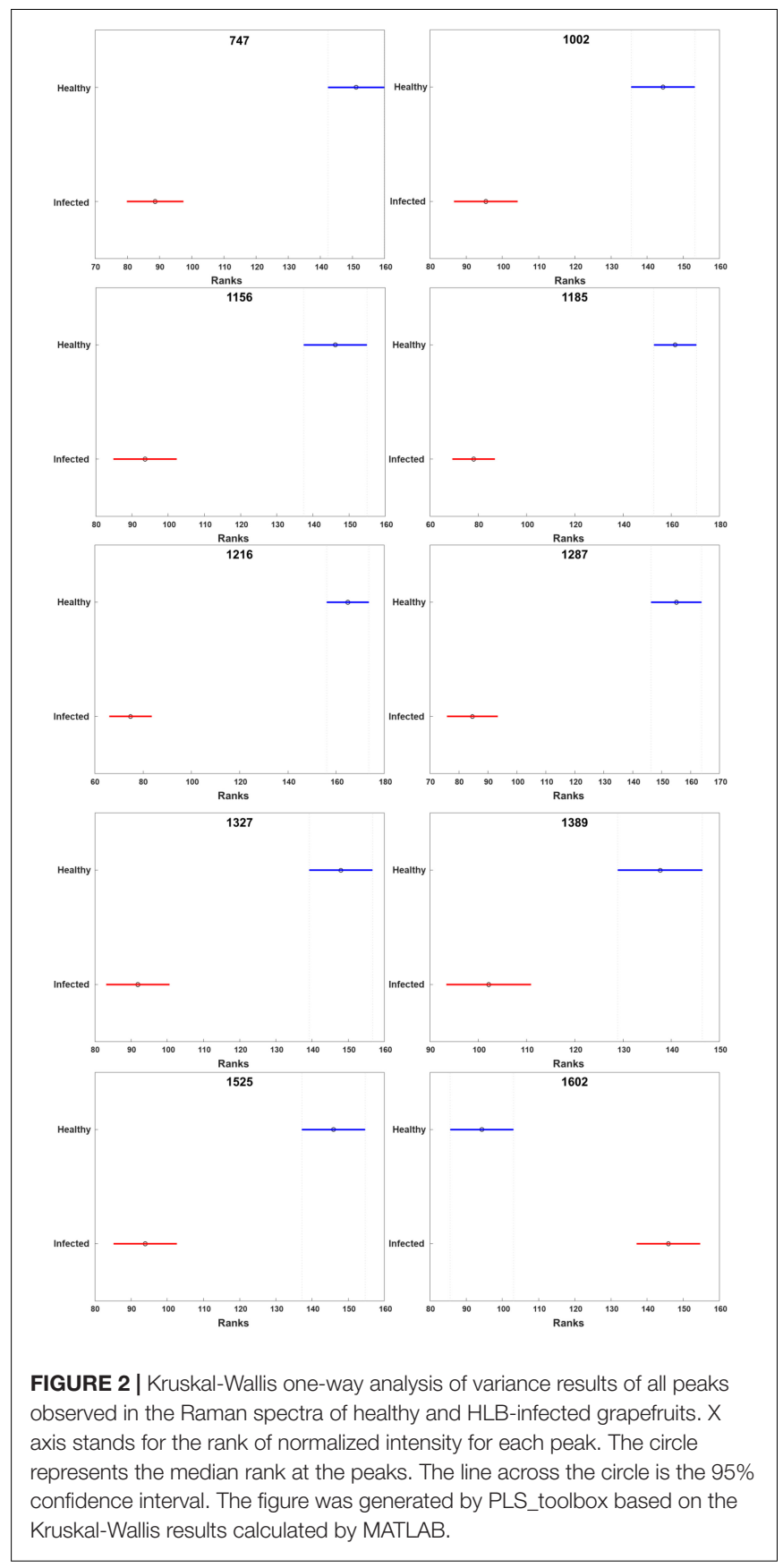

GCACGACAA-3'), and $2 \mu 1$ of plant DNA with an average DNA concentration of $45 \mu \mathrm{M}$; the total reaction volume was scaled to $10 \mu \mathrm{l}$. The $\mathrm{qPCR}$ cycling had one cycle at $95^{\circ} \mathrm{C}$ for $3 \mathrm{~min}$ followed by 39 two-step cycles each at $95^{\circ} \mathrm{C}$ for $15 \mathrm{~s}$ and $55^{\circ} \mathrm{C}$ for $30 \mathrm{~s}$, and a final melting curve of $65-95^{\circ} \mathrm{C}$ for $5 \mathrm{~s}$. Ca. L. asiaticus gene encoding $\beta$-subunit of ribonucleotide reductase (RNR) was amplified to detect Ca. L. asiaticus (Zheng et al., 2016). A citrus GLYCERALDEHYDE-3-PHOSPHATE DEHYDROGENASE C2 (GAPC2) (Mafra et al., 2012) gene was used as the endogenous reference gene for the normalization of qPCR data. The Ca. $L$. asiaticus $\mathrm{Ct}$ (threshold cycle) values were normalized to the citrus

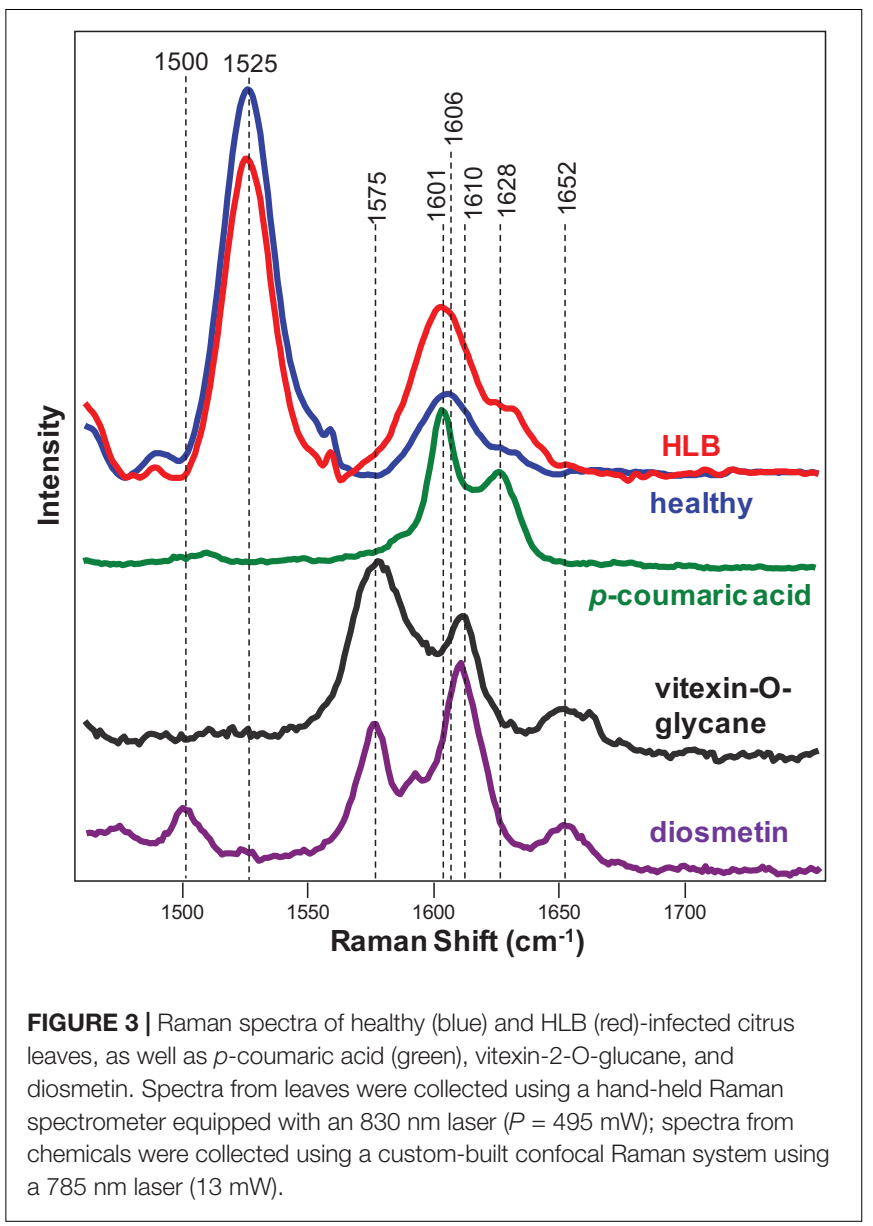

housekeeping gene GAPC2. In order to differentiate healthy from HLB-positive samples, a $\mathrm{Ca}$. $L$. asiaticus $\mathrm{Ct}$ cutoff value $\leq 28$ was used (Supplementary Table 1).

\section{Carotenoid Extraction}

Collected leaf samples were $(\sim 150 \mathrm{mg})$ homogenized using a mortar and pestle. A $1.5 \mathrm{~mL}$ solution of chloroform and dichloromethane $(2: 1, \mathrm{v} / \mathrm{v})$ was added to the homogenate and agitated on a thermomixer at $500 \mathrm{rpm}$ at $4^{\circ} \mathrm{C}$ for $30 \mathrm{~min}$. For phase separation, $0.5 \mathrm{~mL}$ of $1 \mathrm{M}$ sodium chloride solution was added to the homogenate, mixed by inversion, and centrifuged at 5,000 g for $10 \mathrm{~min}$. The aqueous and organic phases were collected in separate tubes. The aqueous phase was subjected to another round of separation by adding $0.75 \mathrm{~mL}$ of chloroform and dichloromethane $(2: 1, \mathrm{v} / \mathrm{v})$, followed by centrifugation at $5,000 \mathrm{~g}$ for $10 \mathrm{~min}$. The second organic phase was collected and pooled with the first batch and dried by the centrifugal evaporation method. Dried pellet was re-dissolved in $200 \mu \mathrm{L}$ of methanol/tert-methyl butyl ether (MTBE) $(60 / 40, \mathrm{v} / \mathrm{v})$ prior to injection into HPLC.

\section{HPLC Analysis}

Leaf extracts were analyzed by reversed phase HPLC using a Waters 1525 pump equipped with a Waters 2707 auto 


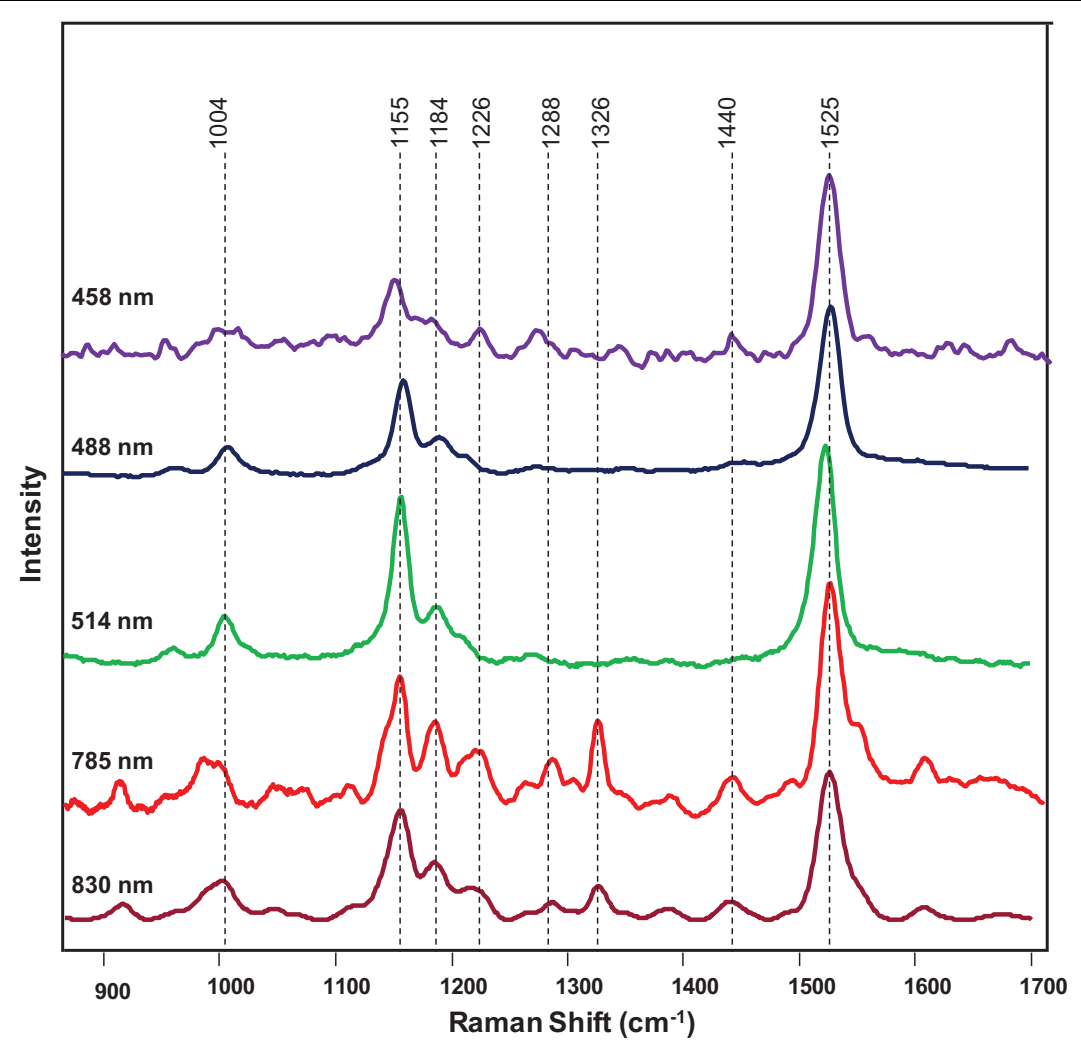

FIGURE 4 | Raman spectra of a citrus leaf collected at 458 nm (purple), 488 nm (navy blue), 514 nm (green), 785 nm (red), and $830 \mathrm{~nm}$ (maroon).

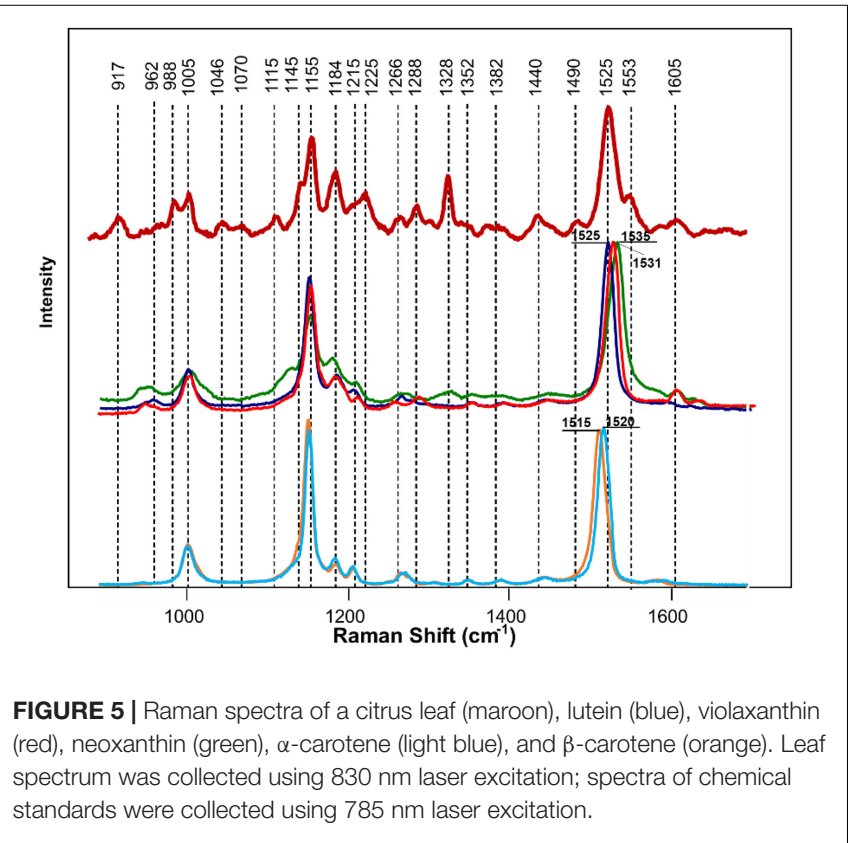

sampler and a Waters 2489 photodiode array detector (PDA). Carotenoids were separated on a reverse-phase $\mathrm{C}_{30}, 3 \mu \mathrm{m}$ column $(250 \times 4.6 \mathrm{~mm})$ (Thermofisher, part number 075723) using mobile phases consisting of (A) methanol/water $(95: 5, \mathrm{v} / \mathrm{v})$ and
(B) MTBE. The gradient elution used with this column was $97 \%$ A and $3 \%$ B at 0-6 min with a linear increase of B to $100 \%$ at $20 \mathrm{~min}$ and was returned to initial conditions at $23 \mathrm{~min}$. The column temperature was maintained at $20^{\circ} \mathrm{C}$. The eluting peaks were monitored at $450 \mathrm{~nm}$ using PDA. Quantification was performed using Breeze software comparing peak area with standard reference curves.

\section{Raman Spectroscopy}

Four Raman spectra were collected from a leaf on the adaxial side using a hand-held Resolve Agilent spectrometer. Spectral acquisition time was $1 \mathrm{~s}$, laser power $495 \mathrm{~mW}$; spectral baseline corrected was performed by the spectrometer software. In total, around 50 surface spectra were collected from both HLB-infected and healthy plants. The Raman spectra of chemical standards were collected using a home-built confocal Raman microscope equipped with a Nikon TE-2000U inverted microscope. Laser light $(\lambda=785 \mathrm{~nm})$ was generated by a solid-state continuous wavelength laser (Necsel, NJ, United States) and directed toward a 50/50 beam splitter (Chroma, Marlborough, MA). The sample was illuminated though $20 \mathrm{X}$ dry Nikon objective $(\mathrm{NA}=0.45)$. The scattered light was collected by the same objective and directed back to the beam splitter. Next, elastically scattered photons were filtered out by an LP02-785RE-25 long-pass filter (Semrock, NY, United States). Inelastically scattered photons were dispersed on a 600 groove $/ \mathrm{mm}$ grating (blazed at $750 \mathrm{~nm}$ ) 


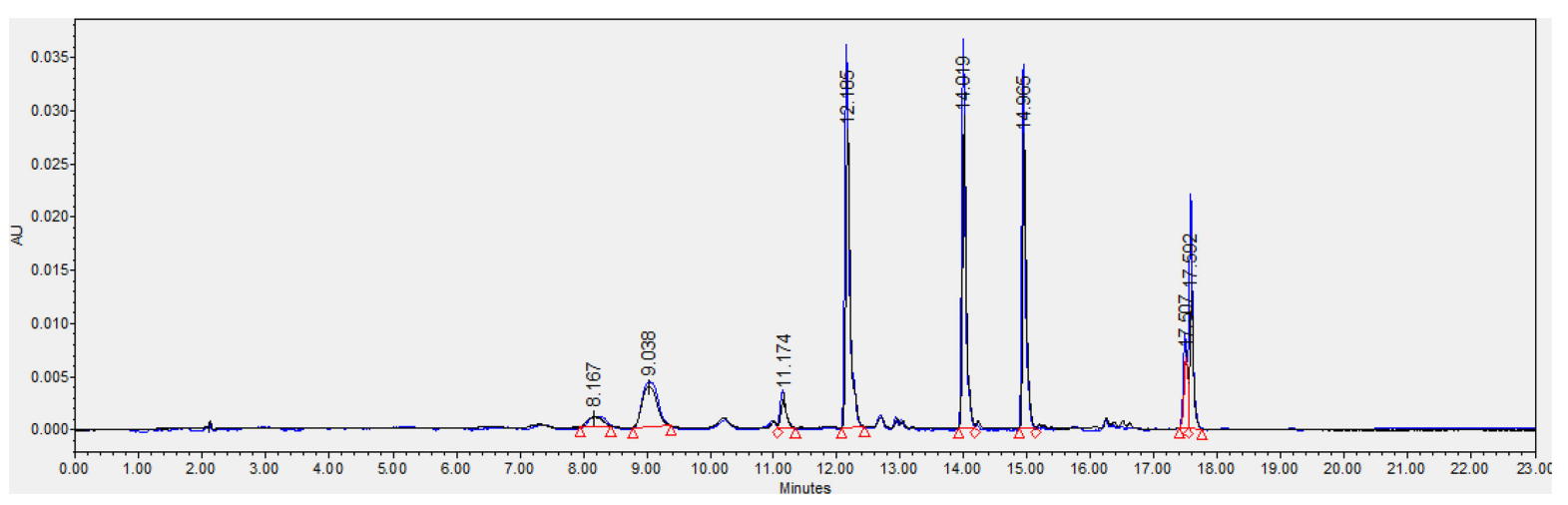

FIGURE 6 | HPLC profiles of healthy (blue) and HLB-infected (black) grapefruit leaves.

in an IsoPlane SCT 320 spectrograph (Princeton Instruments, NJ, United States) and captured using PIXIS:400BR CCD (Princeton Instruments, NJ, United States). Prior Proscan II motorized stage H117P2TE (Prior, MA, United States) was used to control the $\mathrm{XYZ}$ position of the sample. All data were processed using GRAMS/AI 7.0 (Thermo Galactic, NH, United States).

\section{Statistical Analysis}

Raman spectra were imported into MATLAB R2020a (Mathworks) for statistical analysis. Raman spectra were normalized based on overall intensity. Kruskal-Wallis one-way analysis of variance was used to determine the changes at observed bands. Kruskal-Wallis one-way analysis tests whether the median in a set of samples is significantly different from other classes. The null hypothesis of this test was that there was no significant difference at the band of interests. The significant level $(\alpha)$ was 0.05 . The ANOVA also reported a 95\% confidence interval for the true value of median for each compared group. The overlapping confidence intervals were conducted using MATLAB multcompare function, which by default uses Tukey HSD to evaluate group-to-group differences.

\section{RESULTS AND DISCUSSION}

\section{Raman-Based Analysis of HLB and Healthy Plants}

HLB-associated changes in the spectra of citrus trees were in the vibrational bands centered at 1,000,1,155-1,226, and 1,525 $\mathrm{cm}^{-1}$, as well as in 1,601-1,630 $\mathrm{cm}^{-1}$ (Figures 1, 2, Sanchez et al., 2019c). The last two bands originated from polyphenols, also known as phenylpropanoids, large $C a$. L a asiaticus compounds that have aromatic moiety (Agarwal, 2006; Kang et al., 2016). It should be also noted that in the Raman spectrum collected from the leaves of healthy citrus trees, these two bands were centered at 1,606 and $1,630 \mathrm{~cm}^{-1}$, whereas in the spectrum of HLBinfected trees, the bands were found at 1,601 and $1,630 \mathrm{~cm}^{-1}$ (Figures 1, 2 and Supplementary Figure 2). HPLC-MS analysis of HLB-related changes on citrus trees reported by Hijaz and co-authors revealed an increase in the concentration of vitexin-2-O-rhaminozide (flavone), and hydroxycinnamates, also known as p-coumarates (Hijaz et al., 2013). All these $\mathrm{Ca}$. L. asiaticus compounds are phenylpropanoids (Pompeu et al., 2018). Although an increase in the concentration of flavones was small, the concertation of hydroxycinnamates increased more than 10 times (Hijaz et al., 2013). Kruskal-Wallis one-way analysis of variance was conducted to normalized Raman spectra of HLB and healthy leaves. We observed a significant increase at bands at 1,602 $\mathrm{cm}^{-1}$ (Figure 2). This experimental evidence strongly suggests that an increase in the intensities of 1,601 and $1,630 \mathrm{~cm}^{-1}$ (Figure 1) is likely to be caused by an increase in the concentration of $p$-coumarates.

\section{Determination of Spectroscopic Signatures of Molecular Analytes Detected by RS}

Expanding upon this finding, we collected Raman spectra from pure standards of $p$-coumaric acid, as well as two flavones vitexin2-O-rhaminozide and diosmetin (Figure 3). We found that $p$-coumaric acid had two peaks centered at 1,601 and $1,628 \mathrm{~cm}^{-1}$. These results confirm that an increase in the intensity of 1,601

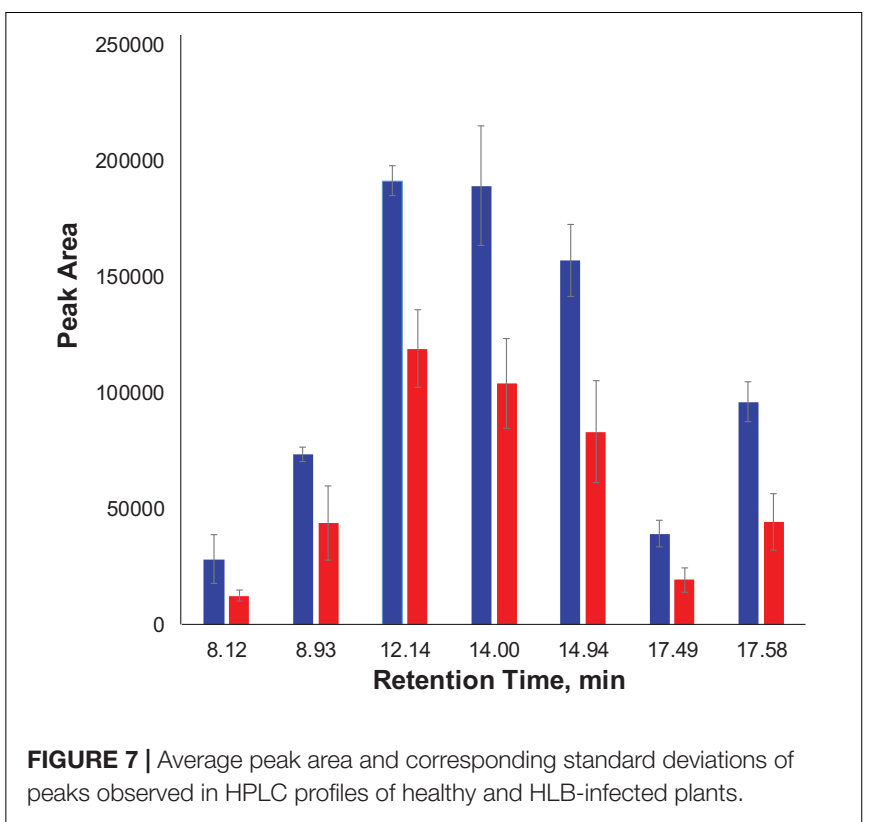


TABLE 1 | Two samples $t$-test results on bands of interests.

\begin{tabular}{|c|c|c|c|c|c|c|c|c|c|c|}
\hline & 747 & 1,002 & 1,156 & 1,185 & 1,216 & 1,287 & 1,327 & 1,389 & 1,525 & 1,602 \\
\hline $\mathrm{H}$ & 1 & 1 & 1 & 1 & 1 & 1 & 1 & 0 & 1 & 1 \\
\hline$P$-value & $2.6140 \mathrm{e}-05$ & $3.5632 \mathrm{e}-08$ & $2.8751 e-09$ & $6.5458 \mathrm{e}-05$ & 0.0014 & 4.3651e-05 & $3.4297 e-06$ & 0.3716 & $1.3999 \mathrm{e}-08$ & $2.1953 e-10$ \\
\hline
\end{tabular}

TABLE 2 | Chromatographic content of HLB-infected and healthy leaves.

\begin{tabular}{lccc}
\hline Retention time & Area & $\%$ Area & Height \\
\hline HLB-infected leaves & & & \\
\hline 8.437 & 27,125 & 8.14 & 876 \\
11.025 & 16,429 & 4.93 & 1,420 \\
12.12 & 101,949 & 30.58 & 14,369 \\
14.000 & 80,566 & 24.16 & 18,123 \\
14.954 & 57,923 & 17.37 & 14,565 \\
17.504 & 14,055 & 4.22 & 3,921 \\
17.589 & 35,366 & 10.61 & 8,633 \\
\hline Healthy leaves & & & \\
\hline 7.546 & 20,148 & 2.87 & 1,037 \\
8.46 & 75,340 & 10.75 & 4,197 \\
12.128 & 186,695 & 26.64 & 35,992 \\
13.994 & 156080 & 22.27 & 35,965 \\
14.953 & 136,928 & 19.54 & 33,854 \\
17.505 & 31,475 & 4.49 & 8,904 \\
17.591 & 94,237 & 13.45 & 22,483 \\
\hline
\end{tabular}

and $1,630 \mathrm{~cm}^{-1}$ bands in the Raman spectra observed upon HLB infection was due to an increase in the concentration of $p$-coumaric acid/p-coumarates in the leaves.

The increase in the $p$-coumaric acid upon HLB infection is also supported by a study by Brodersen et al. (2014). Using microscopy analysis of leaves and stem sections of HLB-infected orange trees, the authors showed deformed cambium cells, collapsed phloem, and increased lignification of parenchyma cells. Indeed, p-coumaric acid is the precursor of H-lignin in plants. Thus, we can suggest that $C a$. L. asiaticus infection triggers synthesis of $p$-coumarates that are further used for $\mathrm{H}$-lignin biosynthesis. The lignification of cell walls likely is a plant defense response against the bacteria in attempts to limit their spread in the infected citrus tree.

In addition to increased intensities of 1,601 and $1,630 \mathrm{~cm}^{-1}$ bands, we observed the appearance of a small shoulder at $1,575 \mathrm{~cm}^{-1}$ in the Raman spectrum collected from the leaves of HLB-infected citrus trees. We also found that both vitexin2-O-rhaminozide and diosmetin exhibited a $1,575 \mathrm{~cm}^{-1}$ band in their spectra (Figure 3). These results suggest that a shoulder at $1,575 \mathrm{~cm}^{-1}$ in the Raman spectrum collected from the leaves of HLB-infected citrus trees is likely to have originated from this flavone. Thus, we can suggest that an increase in the intensity of polyphenol vibrations in the Raman spectrum of HLB-infected trees is due to an increase in the concentration of two compounds: $p$-coumaric acid/ $p$-coumarates and vitexin-2O-rhaminozide and diosmetin.
Next, we investigated the underlying biochemical origin of the observed decrease in the intensities of $1,000,1,155-1,218$, and $1,525 \mathrm{~cm}^{-1}$ vibrations detected in the spectra collected from HLB-infected plants. The bands at 1,000 and $1,525 \mathrm{~cm}^{-1}$ can be assigned to different carotenoid species (Adar, 2017; Devitt et al., 2018), while the vibrations at $1,155,1,184$, and $1,218-1,226 \mathrm{~cm}^{-1}$ could be assigned to cellulose, xylan, or carotenoids (Edwards et al., 1997; Tschirner et al., 2009; Mary et al., 2012; Agarwal, 2014; Kurouski et al., 2015). It is known that carotenoids have absorption maxima in the blue-green part of the electromagnetic spectrum, while cellulose and xylan do not absorb light in that spectral region. Considering the differences in the absorption spectra of carotenoids and carbohydrates, we used a wavelengthscan approach in the blue-green range $(\lambda=458-514 \mathrm{~nm})$ to achieve unambiguous assignment of $1,155,1,184$, and $1,218-$ $1,226 \mathrm{~cm}^{-1}$ vibrations. Our results showed that, under the resonant Raman conditions $(\lambda=458-514 \mathrm{~nm})$, all the bands at $1,005,1,155,1,184,1,215-1,226$, and $1,525 \mathrm{~cm}^{-1}$ could be readily detected in the spectra collected from plant leaves (Figure 4). This suggests that these vibrations originated from the same Ca. $L$. asiaticus biological molecules that are likely to be carotenoids.

\section{Chromatographic and Spectroscopic Analysis of the Carotenoid Profile of Leaves}

We next determined the different carotenoid species that could be assigned to these vibrations. HPLC results of carotenoid analysis in HLB-infected citrus plants performed by Killiny and Nehela revealed a significant decrease in the concentration of chlorophyll a and $b$, pheophytin a, chlorophyllide $a$, violaxanthin, neoxanthin, lutein, isolutein, and $\alpha$ and $\beta$-carotene comparing to healthy plants (Killiny and Nehela, 2017). Our HPLC results (discussed in S1) confirmed these findings (Supplementary Figure 2). Spectroscopic analysis of chlorophyll a revealed that this compound is highly auto-fluorescent and provided no meaningful Raman spectra. Similar results were obtained for its structural analog, chlorophyllin (data not shown). Structural similarity (presence of heme) between chlorophyll b, pheophytin a, and chlorophyllide a from one side and chlorophyll a and chlorophyllin from the other suggests that these compounds do not contribute to the Raman spectrum of the plant leaf. At the same time, Raman spectra of pure standards of $\alpha$ and $\beta$-carotenes, violaxanthin, neoxanthin, and lutein (Figure 5) exhibit similar band patterns to the carotenoid fingerprint of the leaf. We found that a vibrational band around $1,525 \mathrm{~cm}^{-1}$ can be used for identification of carotenoids and, therefore, can be considered as its "marker." Specifically, $\alpha$ and $\beta$-carotenes exhibited this vibration at 1,520 and $1,515 \mathrm{~cm}^{-1}$, respectively. However, we found that in the Raman spectrum of the plant leaf, this band was centered at $1,525 \mathrm{~cm}^{-1}$. This suggests that neither $\alpha\left(1,520 \mathrm{~cm}^{1}\right)$ and $\beta-\left(1,515 \mathrm{~cm}^{1}\right)$ carotenes are likely to contribute to the 
carotenoid signature of the plant leaf. The carotenoid marker was centered at $1,525 \mathrm{~cm}^{-1}$ in the Raman spectra of lutein, whereas in the Raman spectrum of violaxanthin and neoxanthin this band shifted to 1,531 and $1,535 \mathrm{~cm}^{-1}$ individually. Thus, it is likely that lutein is the major carotenoid contributor in the Raman spectrum of the plant leaf.

We also found that violaxanthin has a vibrational band at $\sim 1,605 \mathrm{~cm}^{-1}$ that was not evident in the spectra of other carotenoids. Since the spectroscopic fingerprint of HLB-infected leaves was associated with an increase rather than a decrease of this vibration at $1,605 \mathrm{~cm}^{-1}$ (as discussed above), violaxanthin is unlikely to be the predominant contributor of the carotenoid vibration in HLB plants and that leaves lutein as the primary carotenoid at $1,525 \mathrm{~cm}^{-1}$, that is lowered in HLB-infected leaves. HPLC analysis of grapefruit extracts performed in our laboratory confirmed a major decrease in the concentration of lutein in leaves of HLB-infected grapefruit trees compared to healthy plants (Figures 6, 7 and Table 1). This experimental evidence suggests that RS primarily detects a decrease in the concentration of lutein in plant leaves that take place upon HLB infection. Specifically, we observed a decrease in the concentration of lutein $(\mathrm{RT}=12.185)$, chlorophyll $(\mathrm{RT}=14.019)$, and $\alpha$ and $\beta$-carotene ( $\mathrm{RT}=17.507$ and 17.562 , respectively) in the leaves of HLBinfected plants comparing to healthy grapefruits (Table 2 and Supplementary Table 2).

A decrease in the carotenoid content revealed by both RS and HPLC in the case of HLB infection has strong physiological relevance to plant defense mechanisms (Havaux, 2013). Specifically, biotic and abiotic stresses activate enzymatic oxidation of neoxanthin that yields abscisic acid, a hormone that enhances plant resistance to such stresses (Nambara and MarionPoll, 2005). $\beta$-carotene oxidation and cleavage by reactive oxygen species (ROS) lead to formation of $\beta$-lonone and $\beta$-cyclocitrals that can protect the plant against insects (Nambara and MarionPoll, 2005; Havaux, 2013). Thus, reduction in this molecule could be a consequence of the higher ROS commonly triggered during plant defense reactions (Yu et al., 2007).

\section{CONCLUSION}

Comparative analysis of RS and HPLC/HPLC-MS data of HLBinfected plants enabled characterization of molecular species whose levels were affected during citrus greening. We found that RS detects an increase in p-coumaric acid and several flavones, as well as a decrease in the concentration of lutein in HLB-infected leaves. Our findings suggest that RS can be reliably used to probe various biochemical changes occurring in plants during a defense response. The results of this work, as well as experimental findings reported by other research groups, suggest that RS can be used as a "fast diagnostics" approach that enables rapid screening of plant health (Yeturu et al., 2016; Altangerel et al., 2017; Farber and Kurouski, 2018; Farber et al., 2019b; Mandrile et al., 2019; Sanchez et al., 2019c, 2020b; Gupta et al., 2020; Huang et al., 2020). If more accurate identification of the pathogen strain or species is needed, a molecular methods of analysis, such as PCR, qPCR, or ELISA, can be used. Such a dual diagnostics approach is economically advantageous because RS requires no chemicals; it can be used directly in the field and the results are delivered within seconds. Thus, the use of RS can eliminate a large amount of work for more labor- and time-demanding molecular methods of analyses. We also envision that elucidation of the underlying biochemical determinants of Raman-based diagnostics of biotic and abiotic stresses should enhance the precision of RS. This may eventually eliminate the need for molecular methods of analysis in farming.

\section{DATA AVAILABILITY STATEMENT}

The raw data supporting the conclusions of this article will be made available by the authors, without undue reservation.

\section{AUTHOR CONTRIBUTIONS}

TD, NG, and LS collected spectra. PN performed qPCR analysis of samples. TD performed HPLC and chemometric analyses. SI and PN conducted citrus field surveys, RS sample collection, DNA isolation and qPCR diagnostics to determine HLB status/bacterial titers, and carotenoid extraction. TD, LS, $\mathrm{PN}, \mathrm{KM}$, and DK wrote the manuscript. All authors contributed to the submitted work.

\section{FUNDING}

This study was supported by funds from Texas A\&M AgriLife Research, Texas A\&M University Governor's University Research Initiative (GURI) grant program of (12-2016/M1700437) to DK, and USDA-NIFA-AFRI (2018-70016-28198; HATCH 1023984) and Texas A\&M AgriLife Research Insect-Vectored Disease Seed Grant (124190-96210) to KM.

\section{ACKNOWLEDGMENTS}

We thank Jiaxing Liu (Texas A\&M AgriLife Research) for technical assistance.

\section{SUPPLEMENTARY MATERIAL}

The Supplementary Material for this article can be found online at: https://www.frontiersin.org/articles/10.3389/fpls.2021. 680991/full\#supplementary-material

Supplementary Figure 1 | Average Raman spectra (solid lines) of HLB-infected plants (left) and healthy grapefruits (right) with corresponding standard deviations (dashed lines).

Supplementary Figure 2 | Zoomed spectra of healthy and HLB-infected grapefruit tree leaves.

Supplementary Table 1 | Quantitative real time PCR (qPCR) based diagnostics in healthy and HLB citrus leaves.

Supplementary Table 2 | HPLC identification of carotenoids standard. 


\section{REFERENCES}

Adar, F. (2017). Carotenoids - their resonance raman spectra and how they can be helpful in characterizing a number of biological systems. Spectroscopy 32, 12-20.

Agarwal, U. P. (2006). Raman imaging to investigate ultrastructure and composition of plant cell walls: distribution of lignin and cellulose in black spruce wood (Picea mariana). Planta 224, 1141-1153. doi: 10.1007/s00425006-0295-z

Agarwal, U. P. (2014). 1064 nm FT-Raman spectroscopy for investigations of plant cell walls and other biomass materials. Front. Plant Sci. 5:490. doi: 10.3389/fpls. 2014.00490

Almeida, M. R., Alves, R. S., Nascimbem, L. B., Stephani, R., Poppi, R. J., and De Oliveira, L. F. (2010). Determination of amylose content in starch using Raman spectroscopy and multivariate calibration analysis. Anal. Bioanal. Chem. 397, 2693-2701. doi: 10.1007/s00216-010-3566-2

Almeyda-León, I., Rocha-Peña, M., Soriano, J., and Razo, J. (2001). The use of polymerase chain reaction and molecular hybridization for detection of phytoplasmas in different plant species in Mexico. Revista Mexicana de Fitopatología 19, 1-9.

Altangerel, N., Ariunbold, G. O., Gorman, C., Alkahtani, M. H., Borrego, E. J., Bohlmeyer, D., et al. (2017). In vivo diagnostics of early abiotic plant stress response via Raman spectroscopy. Proc. Natl. Acad. Sci. U S A. 114, 3393-3396. doi: 10.1073/pnas.1701328114

Bové, J. M. (2006). Huanglongbing: a destructive, newly-emerging, century-old disease of citrus. J. Plant Pathol. 88, 7-37.

Brodersen, C., Narciso, C., Reed, M., and Etxeberria, E. (2014). Phloem production in huanglongbing-affected citrus trees. HortScience 49, 59-64. doi: 10.21273/ hortsci.49.1.59

Bueno, J., and Lednev, I. K. (2013). Advanced statistical analysis and discrimination of gunshot residue implementing combined Raman and FT-IR data. Anal. Methods 5, 6292-6296. doi: 10.1039/c3ay40721g

Cantarero, A. (2015). Raman scattering applied to materials science. Procedia Mater. Sci. 9, 113-122. doi: 10.1016/j.mspro.2015.04.014

Capoor, S. P., Rao, D. G., and Viswanath, S. M. (1967). Diaphorina citri Kuway., a vector of the greening disease of citrus in India. Ind. J. Agric. Sci. 37, $572-576$.

Cardona, M. (1975). Light Scattering in Solids. Berlin: Springer-Verlag.

De Moraes Pontes, J. G., Vendramini, P. H., Fernandes, L. S., De Souza, F. H., Pilau, E. J., Eberlin, M. N., et al. (2020). Mass spectrometry imaging as a potential technique for diagnostic of Huanglongbing disease using fast and simple sample preparation. Sci. Rep. 10:13457.

Devitt, G., Howard, K., Mudher, A., and Mahajan, S. (2018). Raman spectroscopy: an emerging tool in neurodegenerative disease research and diagnosis. ACS Chem. Neurosci. 9, 404-420. doi: 10.1021/acschemneuro. $7 \mathrm{~b} 00413$

Edwards, H. G., Farwell, D. W., and Webster, D. (1997). FT Raman microscopy of untreated natural plant fibres. Spectrochim. Acta A Mol. Biomol. Spectrosc. 53A, 2383-2392. doi: 10.1016/s1386-1425(97)00178-9

Egging, V., Nguyen, J., and Kurouski, D. (2018). Detection and identification of fungal infections in intact wheat and sorghum grain using a hand-held raman spectrometer. Anal. Chem. 90, 8616-8621. doi: 10.1021/acs.analchem.8b 01863

Farber, C., and Kurouski, D. (2018). Detection and identification of plant pathogens on maize kernels with a hand-held raman spectrometer. Anal. Chem. 90, 3009-3012. doi: 10.1021/acs.analchem.8b00222

Farber, C., Mahnke, M., Sanchez, L., and Kurouski, D. (2019a). Advanced spectroscopic techniques for plant disease diagnostics. Rev. Trends Analyt. Chem. 118, 43-49. doi: 10.1016/j.trac.2019.05.022

Farber, C., Sanchez, L., and Kurouski, D. (2020). Confirmatory non-invasive and non-destructive identification of poison ivy using a hand-held raman spectrometer. RCS Adv. 10, 21530-21534. doi: 10.1039/d0ra03697h

Farber, C., Shires, M., Ong, K., Byrne, D., and Kurouski, D. (2019b). Raman spectroscopy as an early detection tool for rose rosette infection. Planta 250, 1247-1254. doi: 10.1007/s00425-019-03216-0

Gupta, S., Huang, C. H., Singh, G. P., Park, B. S., Chua, N.-H., and Ram, R. J. (2020). Portable Raman leaf-clip sensor for rapid detection of plant stress. Sci. Rep. 10:20206.
Hager, E., Farber, C., and Kurouski, D. (2018). Forensic identification of urine on cotton and polyester fabric with a hand-held raman spectrometer. Forensic Chem. 9, 44-49. doi: 10.1016/j.forc.2018.05.001

Havaux, M. (2013). Carotenoid oxidation products as stress signals in plants. Plant. J. 79, 597-606. doi: 10.1111/tpj.12386

Hijaz, F. M., Manthey, J. A., Folimonova, S. Y., Davis, C. L., Jones, S. E., and ReyesDe-Corcuera, J. I. (2013). An HPLC-MS characterization of the changes in sweet orange leaf metabolite profile following infection by the bacterial pathogen Candidatus Liberibacter asiaticus. PLoS One 8:e79485. doi: 10.1371/journal. pone.0079485

Huang, C. H., Singh, G. P., Park, S. H., Chua, N. H., Ram, R. J., and Park, B. S. (2020). Early diagnosis and management of nitrogen deficiency in plants utilizing raman spectroscopy. Front. Plant Sci. 11:663. doi: 10.3389/fpls.2020. 00663

Kang, L., Wang, K., Li, X., and Zou, B. (2016). High pressure structural investigation of benzoic acid: raman spectroscopy and x-ray diffraction. J. Phys. Chem. C. 120, 14758-14766. doi: 10.1021/acs.jpcc.6b05001

Killiny, N., and Nehela, Y. (2017). One target, two mechanisms: the impact of 'candidatus liberibacter asiaticus' and its vector, diaphorina citri, on citrus leaf pigments. Mol. Plant Microbe. Interact 30, 543-556. doi: 10.1094/mpmi-02-170045-r

Kurouski, D., Van Duyne, R. P., and Lednev, I. K. (2015). Exploring the structure and formation mechanism of amyloid fibrils by Raman spectroscopy: a review. Analyst 140, 4967-4980. doi: 10.1039/c5an00342c

Kurouski, D., Washington, J., Ozbil, M., Prabhakar, R., Shekhtman, A., and Lednev, I. K. (2012). Disulfide bridges remain intact while native insulin converts into amyloid fibrils. PLoS One 7:e36989. doi: 10.1371/journal.pone. 0036989

Lee, J. A., Halbert, S. E., Dawson, W. O., Robertson, C. J., Keesling, J. E., and Singer, B. H. (2015). Asymptomatic spread of huanglongbing and implications for disease control. Proc. Natl. Acad. Sci. U S A. 112, 7605-7610. doi: 10.1073/ pnas. 1508253112

López-López, M., Delgado, J. J., and García-Ruiz, C. (2013). Analysis of macroscopic gunshot residues by Raman spectroscopy to assess the weapon memory effect. Forensic Sci. Int. 231, 1-5. doi: 10.1016/j.forsciint.2013.03.049

Mafra, V., Kubo, K. S., Alves-Ferreira, M., Ribeiro-Alves, M., Stuart, R. M., Boava, L. P., et al. (2012). Reference genes for accurate transcript normalization in citrus genotypes under different experimental conditions. PLoS One 7:e31263. doi: 10.1371/journal.pone.0031263

Mandrile, L., Rotunno, S., Miozzi, L., Vaira, A. M., Giovannozzi, A. M., Rossi, A. M., et al. (2019). Nondestructive raman spectroscopy as a tool for early detection and discrimination of the infection of tomato plants by two economically important viruses. Anal. Chem. 91, 9025-9031. doi: 10.1021/acs.analchem. $9 \mathrm{~b} 01323$

Mary, Y. S., Panicker, C. Y., and Varghese, H. T. (2012). Vibrational spectroscopic investigations of 4-nitropyrocatechol. Orient. J. Chem. 28, 937-941. doi: 10. $13005 /$ ojc/280239

Mcclean, A. P. D., and Oberholzer, P. C. J. (1965). Citrus psylla, a vector of the greening disease of sweet orange. S. Afr. J. Agric. Sci. 8, 297-298.

Morgan, J. K., Zhou, L., Li, W., Shatters, R. G., Keremane, M., and Duan, Y. P. (2012). Improved real-time PCR detection of 'Candidatus Liberibacter asiaticus' from citrus and psyllid hosts by targeting the intragenic tandem-repeats of its prophage genes. Mol. Cell Probes 26, 90-98. doi: 10.1016/j.mcp.2011.12.001

Nambara, E., and Marion-Poll, A. (2005). Abscisic acid biosynthesis and catabolism. Annu. Rev. Plant Biol. 56, 165-185. doi: 10.1146/annurev.arplant. 56.032604.144046

Pompeu, D. R., Larondelle, Y., Rogez, H., Abbas, O., Pierna, J. A. F., and Baeten, V. (2018). Characterization and discrimination of phenolic compounds using Fourier transform Raman spectroscopy and chemometric tools. Biotechnol. Agron. Soc. Environ. 22, 13-28.

Sanchez, L., Baltensperger, D., and Kurouski, D. (2020a). Raman-based differentiation of hemp. cannabidiol-rich hemp, and cannabis. Anal. Chem. 92, 7733-7737. doi: 10.1021/acs.analchem.0c00828

Sanchez, L., Ermolenkov, A., Biswas, S., Septiningshih, E. M., and Kurouski, D. (2020b). Raman spectroscopy enables non-invasive and confirmatory diagnostics of salinity stresses, nitrogen, phosphorus, and potassium deficiencies in rice. Front. Plant Sci. 11:573321. doi: 10.3389/fpls.2020. 573321 
Sanchez, L., Ermolenkov, A., Tang, X. T., Tamborindeguy, C., and Kurouski, D. (2020c). Non-invasive diagnostics of Liberibacter disease on tomatoes using a hand-held Raman spectrometer. Planta 251:64.

Sanchez, L., Farber, C., Lei, J., Zhu-Salzman, K., and Kurouski, D. (2019a). Noninvasive and nondestructive detection of cowpea bruchid within cowpea seeds with a hand-held raman spectrometer. Anal. Chem. 91, 1733-1737. doi: 10.1021/acs.analchem.8b05555

Sanchez, L., Pant, S., Irey, M. S., Mandadi, K., and Kurouski, D. (2019b). Detection and identification of canker and blight on orange trees using a hand-held raman spectrometer. J. Raman Spectrosc. 50, 1875-1880. doi: 10.1002/jrs. 5741

Sanchez, L., Pant, S., Mandadi, K., and Kurouski, D. (2020d). Raman spectroscopy vs quantitative polymerase chain reaction in early stage huanglongbing diagnostics. Sci. Rep. 10:10101.

Sanchez, L., Pant, S., Xing, Z., Mandadi, K., and Kurouski, D. (2019c). Rapid and noninvasive diagnostics of Huanglongbing and nutrient deficits on citrus trees with a handheld Raman spectrometer. Anal. Bioanal. Chem. 411, 3125-3133. doi: 10.1007/s00216-00019-01776-4

Tsai, J. H., and Liu, Y. H. (2000). Biology of Diaphorina citri (Homoptera:Psyllidae) on four host plants. J. Econ. Entomol. 93, 1721-1725. doi: 10.1603/0022-049393.6.1721

Tschirner, N., Brose, K., Schenderlein, M., Zouni, A., Schlodder, E., Mroginski, M. A., et al. (2009). The anomaly of the $\$ \backslash$ nu $\$ 1$-resonance Raman band of b $\beta$ carotene in solution and in photosystem I and II. Phys. Stat. Sol. 246, 2790-2793. doi: $10.1002 /$ pssb.200982299

Virkler, K., and Lednev, I. K. (2009). Blood species identification for forensic purposes using raman spectroscopy combined with advanced analytical statistics. Anal. Chem. 81, 7773-7777. doi: 10.1021/ac9 01350a

Yeturu, S., Vargas Jentzsch, P., Ciobotă, V., Guerrero, R., Garrido, P., and Ramos, L. A. (2016). Handheld Raman spectroscopy for the early detection of plant diseases: abutilon mosaic virus infecting Abutilon sp. Anal. Methods 8, 34503457. doi: 10.1039/c6ay00381h

Yu, M. M., Schulze, H. G., Jetter, R., Blades, M. W., and Turner, R. F. (2007). Raman microspectroscopic analysis of triterpenoids found in plant cuticles. Appl. Spectrosc. 61, 32-37. doi: 10.1366/00037020777970 1352

Zeng, Z. C., Hu, S., Huang, S. C., Zhang, Y. J., Zhao, W. X., Li, J. F., et al. (2016). Novel electrochemical raman spectroscopy enabled by water immersion objective. Anal. Chem. 88, 9381-9385. doi: 10.1021/acs.analchem.6b0 2739

Zheng, Z., Xu, M., Bao, M., Wu, F., Chen, J., and Deng, X. (2016). Unusual five copies and dual forms of nrdB in "Candidatus Liberibacter asiaticus": Biological implications and PCR detection application. Sci. Rep. 6:39020.

Conflict of Interest: The authors declare that the research was conducted in the absence of any commercial or financial relationships that could be construed as a potential conflict of interest.

Publisher's Note: All claims expressed in this article are solely those of the authors and do not necessarily represent those of their affiliated organizations, or those of the publisher, the editors and the reviewers. Any product that may be evaluated in this article, or claim that may be made by its manufacturer, is not guaranteed or endorsed by the publisher.

Copyright (C) 2021 Dou, Sanchez, Irigoyen, Goff, Niraula, Mandadi and Kurouski. This is an open-access article distributed under the terms of the Creative Commons Attribution License (CC BY). The use, distribution or reproduction in other forums is permitted, provided the original author(s) and the copyright owner(s) are credited and that the original publication in this journal is cited, in accordance with accepted academic practice. No use, distribution or reproduction is permitted which does not comply with these terms. 